\title{
Uncovering the key role of distortion in tetrazine ligations guides the design of bioorthogonal tools with high reactivity and superior stability ${ }^{+}$
}

\section{Introduction}

The inverse electron demand Diels-Alder (IEDDA)-initiated ligation of 1,2,4,5-tetrazines (Tz) with strained dienophiles represents a group of exceptionally fast bioorthogonal reactions..$^{1-3}$ In particular, trans-cyclooctene (TCO) derivatives ${ }^{4-6}$ provide several orders of magnitude higher reactivity than other dienophiles such as cyclopropenes ${ }^{7}$ or norbornenes. ${ }^{8}$ In the rate-determining step the Tz first reacts with TCO in a [4+2]cycloaddition to form a bicyclic intermediate that rapidly undergoes cycloreversion to give dihydropyridazines as ligation products (Fig. 1a). Due to high reaction rates, its biocompatibility, and versatility the Tz/TCO-ligation has found broad application in many fields, in particular enabling selective chemical reactions in living organisms. ${ }^{2,3}$ Very recently, bioorthogonal chemistry has entered Phase 1 clinical trials, with $\mathrm{Tz} / \mathrm{TCO}$-reactions currently being tested in humans, aiming for locally restricted prodrug activation to improve the selectivity of chemotherapeutic treatment of cancer. ${ }^{9,10}$

In recent years, a variety of differently substituted tetrazines has been used for bioorthogonal reactions and in vivo chemistry,

\footnotetext{
a. Institute of Applied Synthetic Chemistry, TU Wien, Getreidemarkt 9, 1060 Vienna, Austria.E-mail: dennis.svatunek@tuwien.ac.at, hannes.mikula@tuwien.ac.at b. Department of Chemistry and Biochemistry, University of California, Los Angeles, Los Angeles, 90095, United States.

\# D. S. and M. W. contributed equally.

† Dedicated to Prof. Johannes Fröhlich on the occasion of his 60th birthday.

Electronic Supplementary Information (ESI) available: Computational methods, organic synthesis, reaction kinetics, compound stability and characterization, copies of NMR spectra; xyz-coordinates of all calculated geometries.

See DOI: $10.1039 / x 0 x x 00000 x$
}

including bis-alkyl-substituted Tz, ${ }^{11-15}$ alkyl-aryl-Tz, ${ }^{16-19}$ monoalkyl-Tz ${ }^{20}$ (alkyl-H-Tz) as well as highly reactive bisheteroary|21,22 and mono-aryl ${ }^{23-26}$ derivatives (aryl-H-Tz). These applications have motivated and fueled the development of advanced procedures for the synthesis of tetrazine scaffolds. ${ }^{27-31}$ In particular, 2-pyridyl-Tz are frequently used despite limited stability, because of their exceptionally high reactivity. This is commonly attributed to the electron-withdrawing effect of the 2-pyridyl substituent, resulting in a lowered orbital energy of the tetrazine, and thereby accelerating the IEDDA cycloaddition. 2,3,32 Assuming that the reactivity is indeed controlled by frontier molecular orbital (FMO) interactions, we hypothesized that 4-pyridyl substituted Tz are even more reactive than their 2-pyridyl analogs. ${ }^{33-35}$

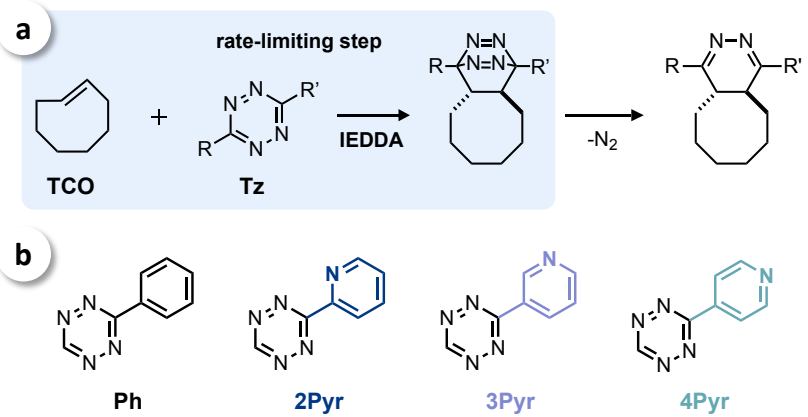

Fig. 1 (a) Inverse electron demand Diels-Alder (IEDDA)-initiated tetrazine (Tz) ligation with trans-cyclooctene (TCO); (b) Selected compounds for the investigation of the impact of different arylsubstituents on Tz reactivity. 
4-Pyridyl-Tz have previously been used for the design of fluorogenic probes. ${ }^{36}$ However, so far there is no comparative data on the IEDDA reaction kinetics of 2-pyridyl- and 4-pyridyltetrazines. Therefore, we selected a series of phenyl (Ph), 2pyridyl (2Pyr), 3-pyridyl (3Pyr), and 4-pyridyl (4Pyr) substituted Tz (Fig. 1b) to study the influence of the aryl substituent on the click reactivity in a combined experimental and computational approach.

\section{Results and discussion}

Theoretical calculations were performed using DFT at the M06-2X-D3/6-311+G(d,p)-SMD(1,4-dioxane)//M06-2X-D3/6-

$31 \mathrm{G}(\mathrm{d}, \mathrm{p})$ level of theory, and orbital energies were calculated at the HF/6-311+G(d,p)//M06-2X-D3/6-31G(d) level of theory. A detailed description of the computational methods used within this study can be found in the Supporting Information. Energies for the reacting LUMO+132 of tetrazines range from 1.35 to $0.95 \mathrm{eV}$. As expected, $\mathbf{P h}$ shows the highest orbital energy followed by $3 \mathbf{P y r}$ and $\mathbf{2 P y r}$, which were calculated to have an almost equal LUMO+1 level, while 4Pyr shows the lowest orbital energy (Fig. 2a). According to FMO theory 4Pyr should thus indeed show an increased IEDDA reactivity compared to 2 Pyr.

To compare these results with measured reaction rates we have prepared all selected mono-substituted aryl-tetrazines using a method recently published by Audebert and coworkers. ${ }^{27}$ Rate constants for the ligation with TCO $^{37}$ were measured by monitoring IEDDA reactions in anhydrous 1,4dioxane at $25^{\circ} \mathrm{C}$ by stopped-flow spectrophotometry. The measured rate constants span an order of magnitude ranging from $100 \mathrm{M}^{-1} \mathrm{~s}^{-1}$ for $\mathbf{P h}$, to $620 \mathrm{M}^{-1} \mathrm{~s}^{-1}$ for $2 \mathrm{Pyr}$ (Fig. 2b). While the reactivity trend for $\mathbf{P h}, \mathbf{3 P y r}$, and $\mathbf{4} \mathbf{P y r}$ seems to be governed by FMO interactions (electron-withdrawing effects), $\mathbf{2 P y r}$ is significantly more reactive ( $>3$-fold) than expected based on the respective orbital energy (Fig. 2c). Qualitatively equivalent results were obtained using calculated Kohn-Sham orbital energies (see Supporting Information, Fig. S1). Hence, the high IEDDA reactivity of $\mathbf{2 P y r}$ cannot be attributed to the electronwithdrawing effect of the 2-pyridyl substituent.
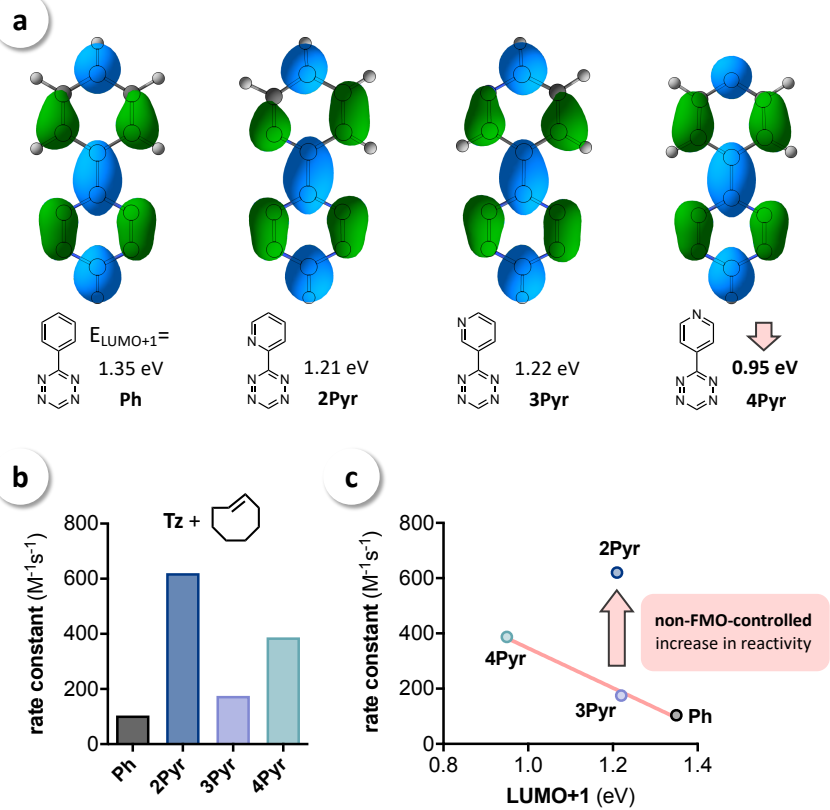

Fig. 2 (a) LUMO+1 orbitals and orbital energies of mono-substituted Tz showing a significantly lower LUMO+1 energy for 4Pyr; (b) Rate constants for the reaction of Ph, 2Pyr, 3Pyr, and 4Pyr with TCO (1,4dioxane, $25{ }^{\circ} \mathrm{C}, \mathrm{n}=6, \mathrm{SD}<1 \%$ ); (c) Measured rate constants vs. calculated LUMO+1 energy.

To investigate the origin of the observed reactivity trend for different pyridyl substituents a DFT study of the respective reactions was performed showing good correlation between the calculated free energies of activation $\left(\Delta G^{\ddagger}\right)$ and the experimental values (Fig. S2). The calculated transition state geometries revealed a highly conserved distance of the forming bond at C-1 of the Tz of $2.33 \pm 0.01 \AA$. However, the distance at C- 4 and thus the asynchronicity differ between the analyzed tetrazine scaffolds (Fig. 3a).

For detailed investigation of the barrier heights we performed a distortion/interaction analysis (also referred to as activation/strain model) ${ }^{38}$ on all four transition states (Fig. 3b) using the autoDIAS software package. ${ }^{39}$ This energy decomposition method was introduced independently by Houk

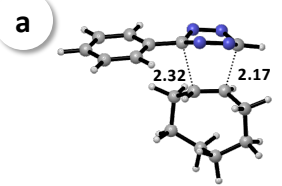

$\Delta \mathbf{G}^{\ddagger}=16.6$

$\mathrm{Ph}+\mathrm{TCO}$

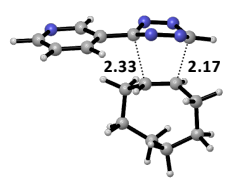

$\Delta \mathbf{G}^{\ddagger}=16.0$

3Pyr + TCO

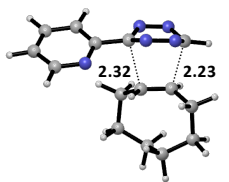

14.8

$2 \mathrm{Pyr}+\mathrm{TCO}$

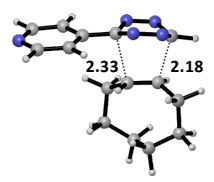

15.5

$4 \mathrm{Pyr}+\mathrm{TCO}$

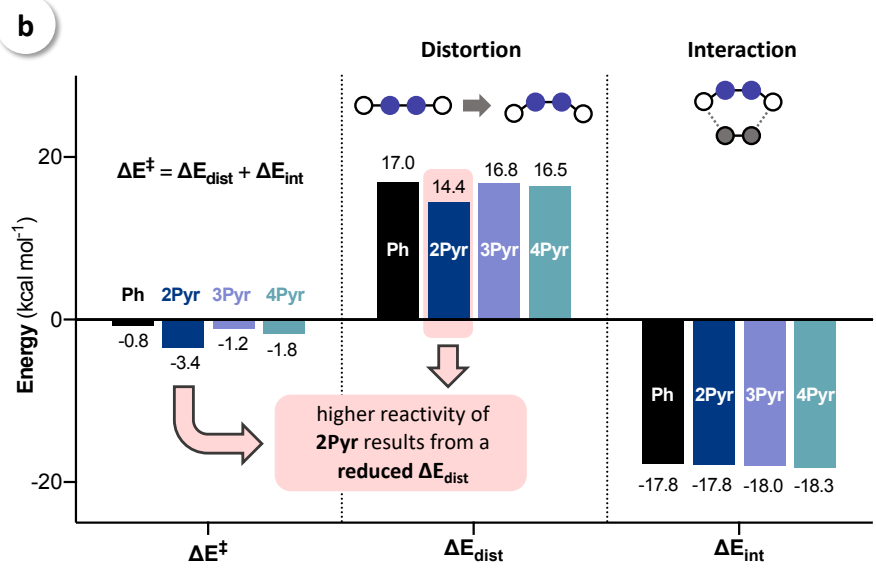

Fig. 3 (a) Calculated transition state geometries and free energies of activation $\left(\Delta \mathrm{G}^{\ddagger}, \mathrm{kcal} \mathrm{mol}^{-1}\right)$ for the reaction of mono-substituted Tz (Ph, 2Pyr, 3Pyr, 4Pyr) and TCO; (b) Distortion/interaction analysis shows that the high reactivity of 2-pyridyl-substituted Tz results from a reduced distortion energy ( $\Delta \mathrm{E}_{\text {dist }}$ ). 
and Bickelhaupt and has successfully been applied to investigate bioorthogonal cycloadditions. ${ }^{32,40-42}$ In this analysis, the energy of activation $\left(\Delta \mathrm{E}^{\ddagger}\right)$ is dissected into two parts, the distortion energy $\left(\Delta \mathrm{E}_{\text {dist }}\right)$, which is needed to distort the isolated reactants into transition state geometry, and the interaction energy $\left(\Delta \mathrm{E}_{\text {int }}\right)$ resulting from bringing the two distorted isolated fragments together. The analysis was performed at the transition state as a defined point for each reaction due to different asynchronicity during the reaction. Interaction energies were calculated to be similar for all studied reactions (within $0.5 \mathrm{kcal} \mathrm{mol}^{-1}$ ) and do not explain the observed differences in IEDDA reactivity. However, $\Delta \mathrm{E}_{\text {dist }}$ for $\mathbf{2} \mathbf{P y r}$ is more than $2 \mathrm{kcal} \mathrm{mol}^{-1}$ lower than for $\mathbf{P h}, 3 \mathbf{P y r}$, and $\mathbf{4 P y r}$ demonstrating that the increased reactivity of $2 \mathrm{Pyr}$ with TCO is caused by a reduced distortion energy (Fig. 3b).

To explain the lowered $\Delta \mathrm{E}_{\text {dist }}$ for aryl substituents with nitrogen atoms in 2-position we have focused on the geometries encountered at the transition state. In all cases the aryl moiety is tilted away from the allylic $\mathrm{CH}_{2}$ of TCO. For $\mathbf{P h}$, 3 Pyr and $\mathbf{4 P y r}$ the dihedral angle is approximately $80^{\circ}$, which we reasoned is due to the steric demand of the allylic $\mathrm{CH}_{2}$. This hypothesis is in agreement with investigations of analogous reactions with ethylene (no allylic $\mathrm{CH}_{2}$ ), showing dihedral angles of approx. 90 (Fig. 4a, Fig. S3). However, for 2 Pyr we observed a much stronger tilt in the transition state for the reaction with TCO, with a dihedral angle of $62^{\circ}$, which did not change in the reaction with ethylene. These observations demonstrate that it is an intrinsic property of 2-pyridyl-substituted tetrazines rather than forced by steric interactions. In fact, the calculated geometry of $\mathbf{2 P y r}$ revealed that a nitrogen-nitrogen interaction destabilizes the reactant. This interaction becomes apparent when looking at the stabilization energies when going from an orthogonal (i.e., dihedral angle between the two aromatic rings of $90^{\circ}$ ) conformer in the reactant to the stable, coplanar conformer. For Ph, 3Pyr and 4Pyr this stabilization energy based on conjugation between the ring systems is $7-8 \mathrm{kcal} \mathrm{mol}^{-1}$. For 2Pyr the most stable conformation is still planar, however, the stabilization is only $5 \mathrm{kcal} \mathrm{mol}^{-1}$ due to a destabilizing interaction between the pyridyl nitrogen and the vicinal Tz nitrogen. At the transition state this $\mathrm{N}-\mathrm{N}$ repulsion can be avoided by rotation of the substituent, thereby increasing the distance between the interacting nitrogen atoms. In addition, the nitrogen lone pairs then point in different directions, further reducing the destabilizing interaction.

Hence, our results revealed that the high reactivity of 2pyridyl-tetrazines cannot be explained by the electronwithdrawing nature of the heteroaryl-substituent only, but moreover by intramolecular $\mathrm{N}-\mathrm{N}$ repulsion, finally uncovering the mechanistic key role of distortion in tetrazine ligations.

To confirm that these findings can be translated to physiological conditions, additional IEDDA reactions were carried out in Dulbecco's phosphate buffered saline (DPBS) at $37{ }^{\circ} \mathrm{C}$. Due to the limited stability of the mono-substituted $\mathrm{H}-$ tetrazines in aqueous solution we have prepared the respective methyl-Tz MePh, Me2Pyr, Me3Pyr, and Me4Pyr, and determined the second order rate constants for the reaction with water-soluble TCO-PEG 4 (Fig. 5a). This TCO derivative has
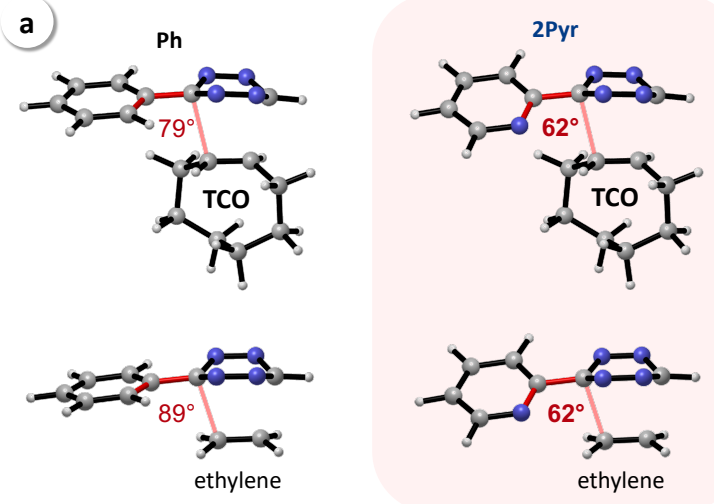

b

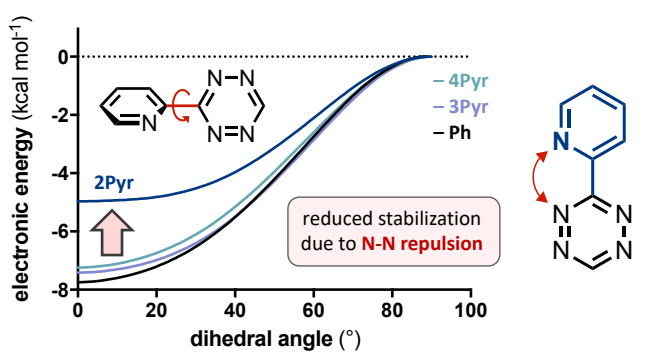

Fig. 4 (a) Dihedral angle in the transition states for the reaction of $\mathbf{P h}$ and 2Pyr with TCO and ethylene (for 3Pyr and 4Pyr see Fig. S2); (b) Calculated energy profiles for the rotation of the aryl-Tz bond showing that $\mathrm{N}-\mathrm{N}$ repulsion reduces the stabilization energy for $\mathbf{2 P y r}$.

been prepared starting from axially configured $\mathrm{TCO}-\mathrm{OH}$, a frequently used tag for the design of in vivo chemical tools. $22,43,44$ The observed relative reactivity profile almost exactly matches the data as previously obtained for the reactions of the corresponding aryl-H-Tz with TCO in 1,4dioxane (cf. Fig. 2b), with Me2Pyr showing the highest rate constant in the reaction with TCO-PEG 4 (Fig. 5a).

It is generally accepted that increasing the reactivity of the tetrazine by using electron-withdrawing substituents leads to reduced stability in aqueous/biological media due to accelerated attack of nucleophiles causing Tz degradation (Fig. 5b). ${ }^{1-3,45,46}$ However, when working with buffered aqueous solutions of the aryl-methyl-Tz we noticed a significantly faster degradation of $\mathbf{4 P y r}$ compared to $\mathbf{2 P y r}$ (as indicated by accelerated fading of the characteristic pink color of Tz), despite the higher reactivity of the 2-pyridyl-Tz. To investigate this observation, MePh, Me2Pyr, Me3Pyr and Me4Pyr were incubated in full cell growth medium (Dulbecco's Modified Eagle's medium, DMEM, incl. 10\% fetal bovine serum, FBS) at $37^{\circ} \mathrm{C}$ and Tz stability was monitored by spectrophotometry (Fig. 5b). Indeed, Me4Pyr degraded much faster (30\% left after 12 hours) compared to Me3Pyr and Me2Pyr (approx. 85\%), and MePh (>95\%). These results correlate well with decreasing LUMO+1 energy ${ }^{32}$ of the Tz (Fig. 5b), which we used as a measure for increased electron-withdrawing of the (hetero)aryl substituent. This result is in agreement with the hypothesis of accelerated nucleophilic attack leading to Tz degradation. Due to distortion Me2Pyr is thus not only more reactive than predicted by FMO theory, but moreover shows a significantly higher stability than expected based on observed reaction rates. 


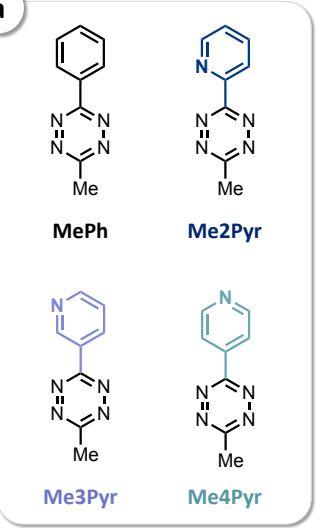

b

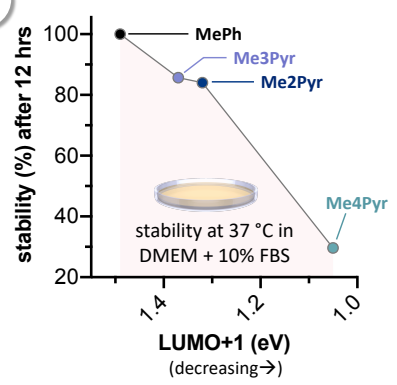

Fig. 5 (a) Reaction kinetics of MePh, Me2Pyr, Me3Pyr and Me4Pyr in buffered aqueous solution (DPBS) at $37^{\circ} \mathrm{C}$ using water-soluble TCO$\mathrm{PEG}_{4}\left(\mathrm{n}=6, \mathrm{SD}<1 \%\right.$ ); (b) Stability of $\mathrm{Tz}$ in cell growth medium at $37^{\circ} \mathrm{C}$ $(n=3, S D<5 \%)$ revealing accelerated degradation with decreasing LUMO+1 energy as a measure for increased electron-withdrawing of the (hetero)aryl substituent, promoting the attack of nucleophiles ( $\mathrm{Nu}$ ).

Motivated by these key mechanistic insights, we aimed to exploit intramolecular $\mathrm{Tz}$ interactions to evade the reactivity/stability trade-off, enabling the design of advanced bioorthogonal tetrazine tools.

Very recently, Joseph Fox and coworkers have reported on the increased IEDDA reactivity of vinyl ether-substituted Tz. ${ }^{29}$ Inspired by these results, we hypothesized that the observed unexpected boost in reactivity is due to lowered distortion energies, caused by intramolecular O-N-repulsion (Fig. 6a). Considering the non-electron-withdrawing character of the vinyl ether-moiety, such scaffolds would thus potentially enable the development of highly reactive tetrazines with substantially increased stability. Computational investigations were thus performed using the Tz structures MVE (methylvinyl ether-Tz) and MV (methylvinyl-Tz). The optimized geometries revealed that an oxygen-nitrogen interaction destabilizes MVE leading to a reduced rotational barrier of $5.2 \mathrm{kcal} \mathrm{mol}^{-1}$ for MVE (similar to 2Pyr) in comparison to $6.7 \mathrm{kcal} \mathrm{mol}^{-1}$ for $\mathbf{M V}$ (Fig. 6b). The calculated LUMO+1 energies moreover indicate a non-electronwithdrawing character of both substituents ( $1.4 \mathrm{eV}$ in contrast to $0.95 \mathrm{eV}$ as calculated for $\mathbf{4 P y r}$ ). The transition state geometries for the reaction with TCO (Fig. 6c) showed a significantly stronger tilt of the vinyl-Tz bond in the case of the vinyl ether-Tz MVE (dihedral angle of $65^{\circ}$ ). The calculated distortion energies $\left(\Delta E_{\text {dist }}\right.$ ) finally confirmed O-N repulsion to be the main reason for the increased reactivity of MVE, as indicated by the calculated free energy of activation $\left(\Delta G^{\ddagger}\right)$.
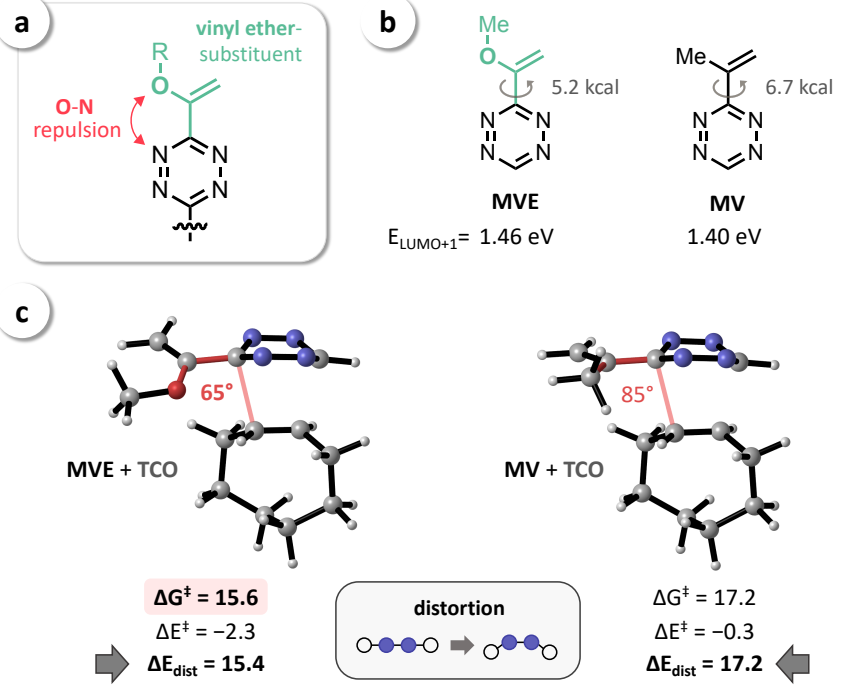

Fig. 6 (a) O-N interaction destabilizes and thereby increases the reactivity of vinyl ether-Tz; (b) Computational analysis revealed a reduced rotational barrier for $\mathbf{M V E}$ in comparison to $\mathbf{M V}$, and a relatively high LUMO+1 energy of $1.46 \mathrm{eV}$; (c) Optimized transition state geometries ( $\mathrm{Tz}+\mathrm{TCO}$ ) and distortion/interaction analysis confirmed $\mathrm{O}$ $\mathrm{N}$ repulsion to be the main reason for the increased reactivity of MVE, as indicated by the calculated values for $\Delta \mathrm{G}^{\ddagger}, \Delta \mathrm{E}^{\ddagger}$ and $\Delta \mathrm{E}_{\text {dist }}\left(\mathrm{kcal} \mathrm{mol}^{-1}\right)$.

Moreover, these results suggest an IEDDA reactivity of MVE similar to $4 \mathrm{Pyr}\left(\Delta \mathrm{G}^{\ddagger}{ }_{\mathrm{MVE}}=15.6 \mathrm{kcal} / \mathrm{mol}\right.$ vs. $\Delta \mathrm{G}_{4 \mathrm{Pyr}}^{\ddagger}=$ $15.5 \mathrm{kcal} / \mathrm{mol})$.

Encouraged by the computational results, we have prepared the vinyl ether-Tz MeEVE and the 3,4-dihydro-2H-pyran (DHP)substituted tetrazines MeDHP and $\mathbf{D H P}_{\mathbf{2}}$ (Fig. 7a; for details on synthetic procedures see the Supporting Information). Second order rate constants for the reactions of these Tz with TCO-PEG in DPBS at $37^{\circ} \mathrm{C}$ were determined by stopped-flow spectrophotometry. As predicted by DFT calculations (vide supra) the IEDDA reactivity of MeEVE (2750 $\mathrm{M}^{-1} \mathrm{~s}^{-1}$ ) indeed perfectly matches the observed value for Me4Pyr $\left(2740 \mathrm{M}^{-1} \mathrm{~s}^{-1}\right)$. In comparison, the cyclic vinyl ether-Tz MeDHP was shown to be less reactive $\left(1820 \mathrm{M}^{-1} \mathrm{~s}^{-1}\right)$, though still significantly faster than the aryl-Tz MePh (990 $\left.\mathrm{M}^{-1} \mathrm{~s}^{-1}\right)$. As expected, we observed a high reactivity of the bis-vinyl ether-Tz $\mathrm{DHP}_{\mathbf{2}}\left(6450 \mathrm{M}^{-1} \mathrm{~s}^{-1}\right)$, exceeding the rate constant of Me2Pyr (5120 $\mathrm{M}^{-1} \mathrm{~s}^{-1}$ ) by approx. 25\% (Fig. 7a). These results finally confirm the distortion-induced IEDDA acceleration due to intramolecular $\mathrm{O}-\mathrm{N}$ repulsion and show that increased reactivities similar to pyridyl-Tz can be achieved by using non-electron-withdrawing vinyl ether substituents.

Subsequent investigation of Tz stability in full cell growth medium at $37{ }^{\circ} \mathrm{C}$ moreover revealed a high stability of vinyl ether-Tz, in particular of MeDHP and $\mathrm{DHP}_{2}$, in contrast to the limited stability of pyridyl-substituted Tz (Fig. 7b). For instance, despite being equal in reactivity, MeDHP is significantly more stable than 4 Pyr (>90\% vs. $<15 \%$ ). Notably, DHP-substituents do not lead to decreased stability, as shown by the data obtained for symmetrical $\mathbf{D H P}_{\mathbf{2}}$ and bis(2-pyridyl)tetrazine (2 $\left.\mathbf{P y r}_{\mathbf{2}}\right)$. While installation of a second DHP had no detrimental effect on stability (>90\% for both MeDHP and $\mathbf{D H P}_{2}$ ), an additional 2pyridyl substituent resulted in almost complete Tz degradation within 24 hours. Despite showing ultrafast IEDDA reaction with 

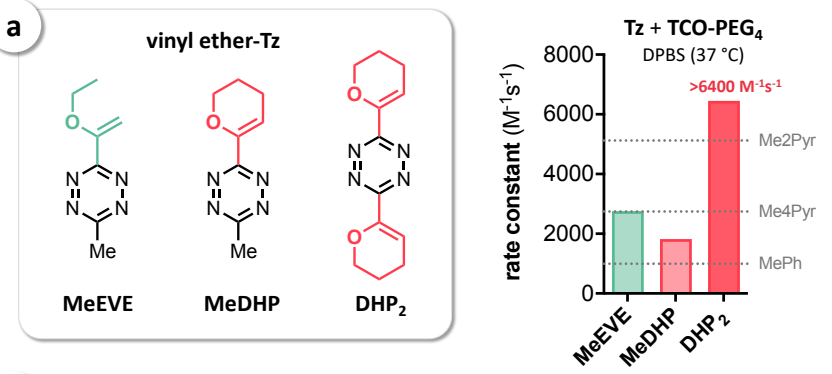

b
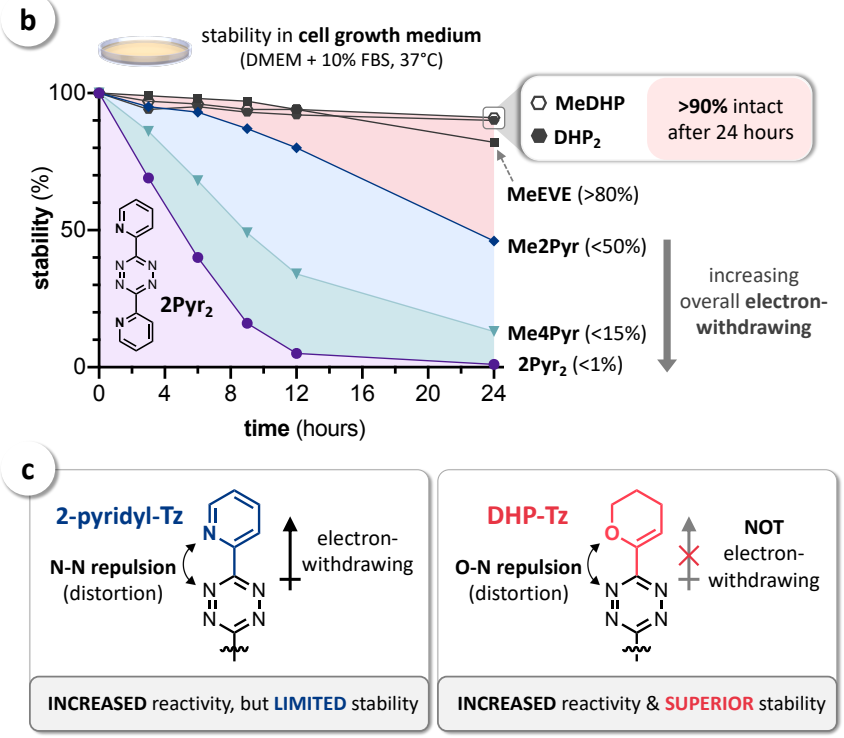

Fig. 7 (a) Vinyl ether-Tz MeEVE, MeDHP and DHP $_{2}$, and second order rate constants for the reaction with TCO-PEG ${ }_{4}$ in buffered aqueous solution (DPBS) at $37^{\circ} \mathrm{C}(\mathrm{n}=6, \mathrm{SD}<1 \%)$; (b) Stability of pyridyl-and vinyl ether-Tz under physiological conditions (full cell growth medium, $37^{\circ} \mathrm{C}$, $\mathrm{n}=3, \mathrm{SD}<5 \%$ ) revealing accelerated degradation of pyridyl-Tz in contrast to the exceptional stability of DHP-substituted Tz; (c) Destabilizing 2-pyridyl-substituents increase the IEDDA reactivity of $\mathrm{Tz}$, also leading to limited stability. In contrast, destabilizing, but nonelectron-withdrawing DHP enables the design of tetrazines with high reactivity and superior stability.

TCO-PEG $4\left(69,400 \mathrm{M}^{-1} \mathrm{~S}^{-1}\right)$, only $<1 \%$ of intact $\mathbf{2} \mathbf{P y r}_{2}$ was detected at the end of the experiment, in comparison to $>90 \%$ of $\mathbf{D H P}_{\mathbf{2}}$ (Fig. 7b). In applications that require extended stability of the $\mathrm{Tz}$ $\left(>10 \mathrm{~h}\right.$ ), $\mathbf{D H} \mathbf{P}_{\mathbf{2}}$ thus outperforms even highly reactive $\mathbf{2} \mathbf{P y r}_{\mathbf{2}}$ (Fig. S4). Overall, these results show that non-electron-withdrawing DHP-substituents can be used to significantly increase the IEDDA reactivity of tetrazines, while also drastically improving compound stability (Fig. 7c).

\section{Conclusions}

Our detailed investigation of the reactivity of pyridyl-Tz uncovered the key role of reduced Tz distortion energies caused by destabilizing intramolecular interactions. Guided by these insights we have been able to show that vinyl ether-substituents increase IEDDA reactivity without accelerating Tz degradation under physiological conditions. In particular, 3,4-dihydro- $2 \mathrm{H}$ pyran (DHP) was identified as a destabilizing, non-electronwithdrawing substituent, enabling the design of tetrazines with high reactivity and superior stability. Finally evading the reactivity/stability trade-off, these findings will thus be instrumental in the development of next-generation bioorthogonal tools, in particular for in vivo chemical strategies that require long-term tetrazine stability. ${ }^{9,47}$

\section{Author Contributions}

D.S. and H.M. conceptualized the study. D.S., M.W., K.H. and H.M. planned the experiments. D.S. performed all calculations. M.W., D.S. and L.H. synthesized and characterized all compounds. M.W. performed reaction kinetics and stability measurements. All authors were involved in the preparation of the manuscript.

\section{Conflicts of interest}

There are no conflicts to declare.

\section{Acknowledgements}

This project has received funding from the European Union's EU Framework Programme for Research and Innovation Horizon 2020, under grant agreement no. 668532. The authors thank Jesper L. Kristensen (University of Copenhagen) for the helpful discussion. The computational results presented have been achieved using the Vienna Scientific cluster (VSC) at TU Wien and the Hoffman2 cluster at UCLA.

\section{Notes and references}

1 S. S. Nguyen and J. A. Prescher, Nat. Rev. Chem., 2020, 4, 476489.

2 S. Mayer and K. Lang, Synthesis, 2017, 49, 830-848.

3 B. L. Oliveira, Z. Guo and G. J. L. Bernardes, Chem. Soc. Rev., 2017, 46, 4895-4950.

4 R. Selvaraj and J. M. Fox, Curr. Opin. Chem. Biol., 2013, 17, 753760.

5 J. E. Pigga, J. E. Rosenberger, A. Jemas, S. J. Boyd, O. Dmitrenko, Y. Xie and J. M. Fox, Angew. Chem. Int. Ed., 2021, 60, 1497514980.

6 A. Darko, S. Wallace, O. Dmitrenko, M. M. Machovina, R. A. Mehl, J. W. Chin and J. M. Fox, Chem. Sci., 2014, 5, 3770-3776.

7 J. Yang, J. Šečkutè, C. M. Cole and N. K. Devaraj, Angew. Chem. Int. Ed., 2012, 51, 7476-7479.

8 A.-C. Knall, M. Hollauf and C. Slugovc, Tetrahedron Lett., 2014, 55, 4763-4766.

9 K. Wu, N. A. Yee, S. Srinivasan, A. Mahmoodi, M. Zakharian, J. M. Mejia Oneto and M. Royzen, Chem. Sci., 2021, 12, 1259-1271.

10 S. Srinivasan, N. A. Yee, K. Wu, M. Zakharian, A. Mahmoodi, M. Royzen and J. M. Mejía Oneto, Adv. Ther., 2021, 4, 2000243.

11 C. Denk, D. Svatunek, T. Filip, T. Wanek, D. Lumpi, J. Fröhlich, C. Kuntner and H. Mikula, Angew. Chem. Int. Ed., 2014, 53, 96559659.

12 C. Denk, D. Svatunek, S. Mairinger, J. Stanek, T. Filip, D. Matscheko, C. Kuntner, T. Wanek and H. Mikula, Bioconjugate Chem., 2016, 27, 1707-1712.

13 R. M. Versteegen, R. Rossin, W. ten Hoeve, H. M. Janssen and M. S. Robillard, Angew. Chem. Int. Ed., 2013, 52, 14112-14116. 
14 J. C. T. Carlson, H. Mikula and R. Weissleder, J. Am. Chem. Soc., 2018, 140, 3603-3612.

15 R. Rossin, R. M. Versteegen, J. Wu, A. Khasanov, H. J. Wessels, E. J. Steenbergen, W. ten Hoeve, H. M. Janssen, A. H. A. M. van Onzen, P. J. Hudson and M. S. Robillard, Nat. Comm., 2018, 9, 1484.

16 X. Fan, Y. Ge, F. Lin, Y. Yang, G. Zhang, W. S. C. Ngai, Z. Lin, S. Zheng, J. Wang, J. Zhao, J. Li and P. R. Chen, Angew. Chem. Int. Ed., 2016, 55, 14046-14050.

17 P. Werther, K. Yserentant, F. Braun, N. Kaltwasser, C. Popp, M. Baalmann, D.-P. Herten and R. Wombacher, Angew. Chem. Int. Ed., 2020, 59, 804-810.

18 J. Tu, D. Svatunek, S. Parvez, A. C. Liu, B. J. Levandowski, H. J. Eckvahl, R. T. Peterson, K. N. Houk and R. M. Franzini, Angew. Chem. Int. Ed., 2019, 58, 9043-9048.

19 A. J. C. Sarris, T. Hansen, M. A. R. de Geus, E. Maurits, W. Doelman, H. S. Overkleeft, J. D. C. Codée, D. V. Filippov and S. I. van Kasteren, Chem. Eur. J., 2018, 24, 18075-18081.

20 M. Wilkovitsch, M. Haider, B. Sohr, B. Herrmann, J. Klubnick, R. Weissleder, J. C. T. Carlson and H. Mikula, J. Am. Chem. Soc., 2020, 142, 19132-19141.

21 R. Rossin, P. Renart Verkerk, S. M. van den Bosch, R. C. M. Vulders, I. Verel, J. Lub and M. S. Robillard, Angew. Chem. Int. Ed., 2010, 49, 3375-3378.

22 E. J. L. Stéen, J. T. Jørgensen, C. Denk, U. M. Battisti, K. Nørregaard, P. E. Edem, K. Bratteby, V. Shalgunov, M. Wilkovitsch, D. Svatunek, C. B. M. Poulie, L. Hvass, M. Simón, T. Wanek, R. Rossin, M. Robillard, J. L. Kristensen, H. Mikula, A. Kjaer and M. M. Herth, ACS Pharmacol. Transl. Sci., 2021, DOI: 10.1021/acsptsci.1c00007.

23 L. G. Meimetis, J. C. T. Carlson, R. J. Giedt, R. H. Kohler and R. Weissleder, Angew. Chem. Int. Ed., 2014, 53, 7531-7534.

24 B. M. Zeglis, K. K. Sevak, T. Reiner, P. Mohindra, S. D. Carlin, P. Zanzonico, R. Weissleder and J. S. Lewis, J. Nucl. Med., 2013, 54, 1389-1396.

25 C. Denk, M. Wilkovitsch, E. Aneheim, M. M. Herth, H. Jensen, S. Lindegren and H. Mikula, ChemPlusChem, 2019, 84, 775-778.

26 R. Garcia-Vazquez, U. M. Battisti, J. T. Jørgensen, V. Shalgunov, L. Hvass, D. L. Stares, I. Nymann Petersen, F. Crestey, A. Löffler, D. Svatunek, J. L. Kristensen, H. Mikula, A. Kjaer and M. M. Herth, Chem. Sci., 2021, in press, https://doi.org/10.1039/D1SC02789A.

27 Y. Qu, F.-X. Sauvage, G. Clavier, F. Miomandre and P. Audebert, Angew. Chem. Int. Ed., 2018, 57, 12057-12061.

28 J. Yang, M. R. Karver, W. Li, S. Sahu and N. K. Devaraj, Angew. Chem. Int. Ed., 2012, 51, 5222-5225.

29 Y. Xie, Y. Fang, Z. Huang, A. M. Tallon, C. W. am Ende and J. M. Fox, Angew. Chem. Int. Ed., 2020, 59, 16967-16973.

30 W. Mao, W. Shi, J. Li, D. Su, X. Wang, L. Zhang, L. Pan, X. Wu and H. Wu, Angew. Chem. Int. Ed., 2019, 58, 1106-1109.

31 W. D. Lambert, Y. Fang, S. Mahapatra, Z. Huang, C. W. am Ende and J. M. Fox, J. Am. Chem. Soc., 2019, 141, 17068-17074.

32 F. Liu, Y. Liang and K. N. Houk, J. Am. Chem. Soc., 2014, 136, 11483-11493.

33 Y.-H. Shin, H.-J. Koh and I.-H. Um, Bull. Korean Chem. Soc., 2017, 38, 1138-1142.

34 C. Hansch, A. Leo and R. W. Taft, Chem. Rev., 1991, 91, 165-195.

35 T. Eicher, S. Hauptmann and A. Speicher, in The Chemistry of Heterocycles, 2nd ed. (Eds.: T. Eicher, S. Hauptmann, A. Speicher), Wiley-VCH, Weinheim, Germany, 2003, pp. 257-310.

36 A. Vázquez, R. Dzijak, M. Dračínský, R. Rampmaier, S. J. Siegl and M. Vrabel, Angew. Chem. Int. Ed., 2017, 56, 1334-1337.
37 D. Svatunek, C. Denk, V. Rosecker, B. Sohr, C. Hametner, G. Allmaier, J. Frohlich and H. Mikula, Monatsh. Chem., 2016, 147, 579-585.

38 F. M. Bickelhaupt and K. N. Houk, Angew. Chem. Int. Ed., 2017, 56, 10070-10086.

39 D. Svatunek and K. N. Houk, J. Comput. Chem., 2019, 40, 25092515.

40 D. Svatunek, N. Houszka, T. A. Hamlin, F. M. Bickelhaupt and H. Mikula, Chem. Eur. J., 2019, 25, 754-758.

41 B. Gold, M. R. Aronoff and R. T. Raines, J. Org. Chem., 2016, 81, 5998-6006.

42 Y. Liang, J. L. Mackey, S. A. Lopez, F. Liu and K. N. Houk, J. Am. Chem. Soc., 2012, 134, 17904-17907.

43 R. Rossin, S. M. J. van Duijnhoven, T. Läppchen, S. M. van den Bosch and M. S. Robillard, Mol. Pharm., 2014, 11, 3090-3096.

44 R. Rossin, S. M. van den Bosch, W. ten Hoeve, M. Carvelli, R. M. Versteegen, J. Lub and M. S. Robillard, Bioconjugate Chem., 2013, 24, 1210-1217.

45 S. G. Tolshchina, G. L. Rusinov and V. N. Charushin, Chem. Heterocycl. Compd., 2013, 49, 66-91.

46 M. R. Karver, R. Weissleder and S. A. Hilderbrand, Bioconjugate Chem., 2011, 22, 2263-2270.

47 A. H. A. M. van Onzen, R. M. Versteegen, F. J. M. Hoeben, I. A. W. Filot, R. Rossin, T. Zhu, J. Wu, P. J. Hudson, H. M. Janssen, W. ten Hoeve and M. S. Robillard, J. Am. Chem. Soc., 2020, 142, 1095510963. 